\title{
2 of 3 dimensional rating scales were useful for discriminating anxiety and depression in adolescents
}

Dierker LC, Albano AM, Clarke GN, et al. Screening for anxiety and depression in early adolescence.J Am Acad Child Adolesc Psychiatry 2001 Aug;40:929-36.

Sources of funding: The John $D$ and Catherine T

MacArthur Foundation and the Health

Research Network on

Development and

Psychopathology.

For correspondence:

Dr L C Dierker,

Wesleyan University,

Middletown, CT, USA.

ldierker@wesleyan.edu
QUESTION: In grade 9 adolescents, what is the diagnostic and discriminative accuracy of 3 dimensional rating scales for detecting anxiety and depressive disorders?
Design

Blinded comparison of 3 rating scales with a DSM-IV diagnosis.

\section{Setting} tucky; and Philadelphia, Pennsylvania, USA.
5 high schools in Portland, Oregon; Louisville, Ken-

Areas under the receiver operating characteristic curve for 3 rating scales*

\begin{tabular}{llll} 
Diagnosis (number of participants) & CES-D & RCMAS & MASC \\
Major depression in girls $(n=8)$ & 0.73 & 0.73 & 0.64 \\
\hline Generalised anxiety disorder in girls $(n=11)$ & 0.52 & 0.62 & 0.82 \\
\hline Social phobia in boys $(n=10)$ & 0.63 & 0.61 & 0.61 \\
\hline Social phobia in girls $(n=21)$ & 0.55 & 0.58 & 0.69 \\
\hline Specific phobia in boys $(n=6)$ & 0.54 & 0.67 & 0.66 \\
\hline Specific phobia in girls $(n=16)$ & 0.56 & 0.51 & 0.60 \\
\hline
\end{tabular}

${ }^{*} \mathrm{CES}-\mathrm{D}=$ Center for Epidemiologic Studies-Depression Scale; RCMAS=Revised Children's Manifest Anxiety Scale; MASC=Multidimensional Anxiety Scale for Children.

\section{COMMENTARY}

Designing brief, effective screening instruments for internalising disorders of childhood has been fraught with difficulty. Correspondence between informants is only fair in these conditions, ${ }^{1}$ so self reports of anxiety or depression do not always imply diagnoses. Showing moderate diagnostic accuracy for 2 such instruments, as Dierker et al do, is thus quite impressive. Although limited by a narrow age range, their findings may pave the way for effective screening in the service of early community intervention programmes. Whether these instruments are also predictive of response to early intervention remains to be examined.

Nevertheless, clinicians should view these instruments as adjunctive to a thorough psychiatric assessment. Although more time consuming, a structured diagnostic interview is still the "gold standard". In younger children, internalising disorders are also difficult to diagnose without corroborating information from parents or other significant adults. Screening instruments can, however, be helpful in monitoring treatment related changes in symptomatology.

The lack of discrimination between disorders on the RCMAS may relate, in part, to the "yes/no" format of this instrument (the other 2 use a Likert type scale). The number of "yes" responses may be more reflective of the respondent's general psychological distress than of any one disorder. Discrimination between disorders on the MASC and CES-D, on the other hand, might have been greater had the authors examined subscale rather than composite scores. Generalised anxiety disorder and comorbid anxiety disorders, for example, would be expected to affect several subscales of the MASC and hence affect the overall composite scores (as they did). Disorders affecting only one subscale would be less likely to be reflected in composite scores.

Finally, the authors' struggle to recruit "high scoring" adolescents for diagnostic interviews when this was linked to participation in a treatment study is noteworthy. Although every effort was made to ensure the sample was representative, their difficulty reflects the need for adolescents to be approached for mental health intervention gradually, in a manner that does not threaten their emerging autonomy.

Katharina Manassis, MD, $\operatorname{FRCP}(\mathrm{C})$

Hospital for Sick Children, Toronto, Ontario, Canada

1 Klein RG. Parent-child agreement in clinical assessment of anxiety and other psychopathology: a review. J Anxiety Disord 1991;5:187-98.

\section{Participants}

632 adolescents (mean age 14 y, $55 \%$ girls) who were in grade 9. Participation was offered to those who scored above the $80^{\text {th }}$ percentile on $\geqslant 1$ of 3 rating scales $(25 \%$ agreed) and a random sample of adolescents who scored below the $80^{\text {th }}$ percentile $(75 \%$ agreed $) .72$ adolescents received the rating scales and the diagnostic standard.

\section{Description of tests and diagnostic standard}

The 3 rating scales were the Center for Epidemiologic Studies-Depression Scale (CES-D), the Revised Children's Manifest Anxiety Scale (RCMAS), and the Multidimensional Anxiety Scale for Children (MASC). The diagnostic standard was a diagnostic interview using the $D S M-I V$ criteria for anxiety and depression. The Anxiety Disorders Interview Schedule for Children was used to assess anxiety, and the Schedule for Affective Disorders and Schizophrenia for School-Age Children was used to assess depression.

\section{Main outcome measures}

Association between diagnosis and rating scale score and area under the receiver operating characteristic (ROC) curve for each scale separately for boys and girls.

\section{Main results}

The table shows the number of participants with each diagnosis. The CES-D composite score was associated with a diagnosis of major depression in girls $(\mathrm{p}<0.05)$. The MASC composite score was associated with a diagnosis of generalised anxiety disorder in girls $(p<0.01)$. Based on ROC curves, the CES-D and the RCMAS were moderately accurate for detecting major depression in girls; the MASC was moderately accurate for detecting generalised anxiety disorder in girls (table). All 3 scales had low accuracy for detecting social phobia and specific phobia, although the MASC had moderate accuracy for detecting children with comorbid social and specific phobia (area under ROC curve 0.73) and girls with comorbid social phobia and generalised anxiety disorder (area under ROC curve 0.80).

\section{Conclusions}

In adolescents, associations existed between the Multidimensional Anxiety Scale for Children score and a diagnosis of anxiety disorder in girls, and between the Center for Epidemiologic Studies-Depression Scale score and a diagnosis for major depression in girls. The Revised Children's Manifest Anxiety Scale score was least successful in discriminating anxiety and depression. 